\title{
Assessment of Measurement of Children's Bone Age Ultrasonically with Sunlight BonAge
}

\author{
Naoto Shimura ${ }^{1}$, Satomi Koyama1 ${ }^{1}$, Osamu Arisaka1, Mariko Imataka ${ }^{1}$, \\ Koshi Sato ${ }^{2}$ and Michiko Matsuura ${ }^{2}$ \\ ${ }^{1}$ Department of Paediatrics Dokkyo University School of Medicine, Tochigi, \\ ${ }^{2}$ Division of Orthodontics and Dentofacial Orthopedics, Graduate School of Dentistry, Tohoku University, \\ Sendai, Japan
}

\begin{abstract}
In this study we measured bone age ultrasonically with Sunlight BonAge (SBA) and report the results of comparisons with radiographs. The subjects were 37 children, who examined at the Pediatric Department of Dokkyo University Hospital, for whom it was thought necessary to measure bone age because of suspicions of body height and pubertal abnormalities. The measurements of bone age were made with SBA during examinations in the outpatient department. In addition, one experienced physician interpreted bone age by the Japanese standard TW2 method (RUS method) and the CASMAS from radiographs taken of their left hands. The results of SBA and TW2 (RUS), and SBA and CASMAS estimations correlated well $(\mathrm{r}=0.89, \mathrm{r}=0.85)$. The inter-measurer error and inter-measurement error were $0.54 \pm 0.36 \mathrm{yr}$ and $0.51 \pm 0.42 \mathrm{yr}$, respectively. SBA calculates bone maturity by an algorithm based on measurements of the width (distance) and speed of sound (SOS) in bones in the wrist. The results of this study show good correlations between the data obtained by SBA and both the RUS method and the CASMAS method. The SBA ultrasound-based skeletal development measurement device is non-invasive, quick and accurate at assessing the bone age of children aged 5 to $15 \mathrm{yr}$ old.
\end{abstract}

Key words: bone age, Sunlight BonAge, ultrasound, CASMAS, TW2

\section{Introduction}

Bone age measurements are an important marker in the management and treatment of endocrine diseases in children and for evaluating their development. Radiographs of the hand are generally used to evaluate bone age, but bone age has to be interpreted based on the appearance and development of the bones (1-3). In this study we

Correspondence: Dr. Naoto Shimura, 880 Kitakobayashi Mibu-cho, Shimotsuga-gun, Tochigi-ken 321-0293, Japan E-mail: nshim@dokkyomed.ac.jp measured bone age ultrasonically with BonAge (Sunlight Medical Ltd., Israel) and report the results of comparisons with radiographs.

\section{Subjects}

The subjects were 37 children (19 boys and 18 girls), aged 5 to $16 \mathrm{yr} 9$ mo (mean $10 \pm 2.9 \mathrm{yr}$ ), who were examined at the Pediatric Department of Dokkyo University Hospital. It was thought necessary to measure their bone age because of suspicions of body height and pubertal abnormalities. There were 11 of short stature, 8 


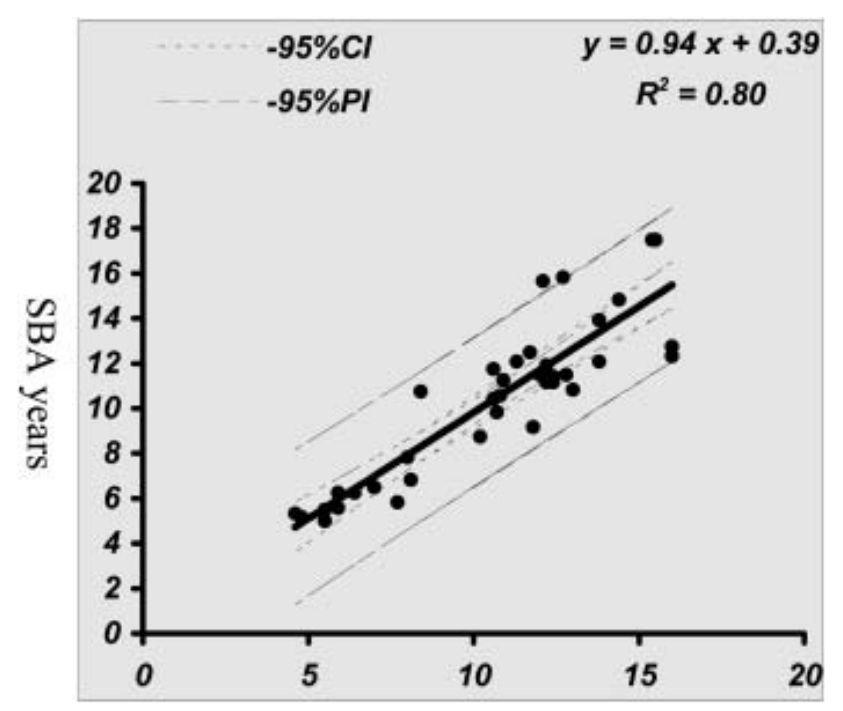

TW2(RUS) years

Fig. 1 TW2 (RUS) and BonAge correlation.

cases of precocious puberty, 6 cases of type 1 diabetes mellitus, 2 cases of growth hormone deficiency, 2 cases of hypopituitarism, and one case each of premature theralche, congenital adrenal hypoplasia, pseudohypoparathyroidism, obesity, type 2 diabetes mellitus, Turner female, anorexia nervosa and fibrous dysplasia.

\section{Methods}

The measurements of bone age were made with Sunlight BonAge (SBA) during examinations in the outpatient department. The measurement site, the head of ulna of the patient's wrist, was marked and the patient's arm was placed in the BonAge measurement unit appropriately; then measurement was performed 5 to 11 times. In addition, one experienced physician interpreted bone age by the Japanese standard TW2 method (RUS method) from radiographs taken of their left hands. The physician also used the same radiographs to measure bone age by the CASMAS method, and both were compared with the results obtained ultrasonically by SBA. Statistical analysis was performed by linear regression and

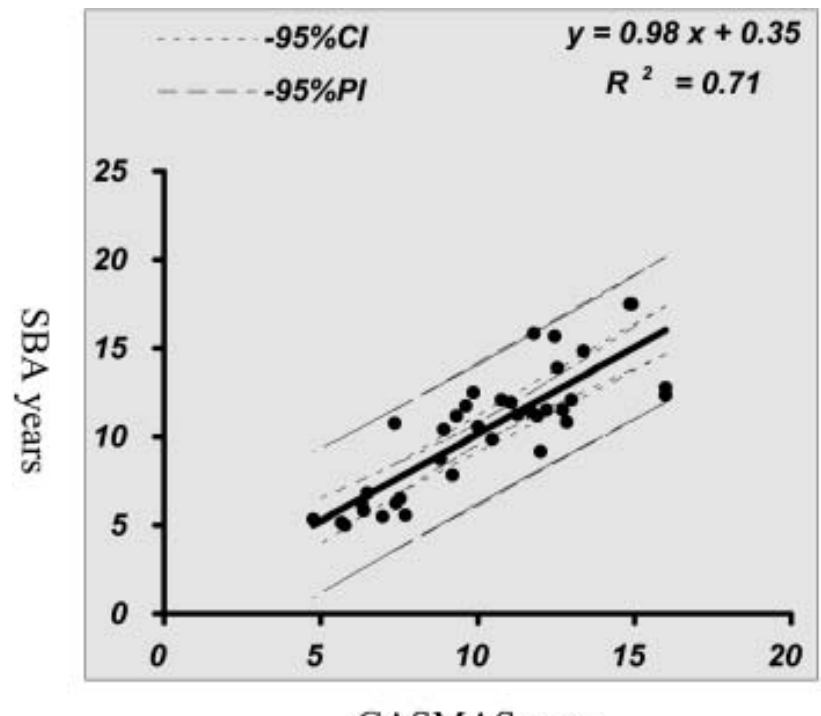

CASMAS years

Fig. 2 CASMAS and BonAge correlation.

Spearman's correlation.

\section{Results}

The linear regression curve and correlation coefficient for the RUS method and SBA was: $\mathrm{y}=$ $0.94 \mathrm{x}+0.39, \mathrm{r}=0.89(\mathrm{p}<0.01)$ (Fig. 1); and for the CASMAS method and SBA: $y=0.98 x+0.35, r=0.85$ $(p<0.01)$ (Fig. 2). The inter-measurer error and inter-measurement error were $0.54 \pm 0.36 \mathrm{yr}$ and $0.51 \pm 0.42 \mathrm{yr}$, respectively.

\section{Discussion}

Recently, sonographic measurement has broadly advanced in areas such as bone density, but sonographic skeletal maturity measurement has not been widely adopted. CastriotaScanderbeg et al. (5) demonstrated the ability of bone maturity assessment using sonographic evaluation of the thickness of the femoral head articular cartilage. The femoral head articular cartilage thickness is assessed and then translated to bone maturity using values obtained from a healthy Italian population (6). 
The Sunlight BonAge is comprised of a main unit and a measurement unit, which has an ultrasound probe with two ultrasound transducers. There is an armrest between the transducers to fixate and support the patient's hand and wrist. During measurement, ultrasonic waves are passed through the patient's left wrist via attached transducers. Then, SBA calculates bone maturity by an algorithm based on measurements of the width (D: distance) and speed of sound (SOS) in bones in the wrist.

In the course of development, children's bones undergo ossification, changing from cartilage to calcified bone. In the case of long bones such as the radius and the ulna, the center part of the bone (diaphysis) is already calcified at birth, but the ends of the bone (epiphysis) are still comprised of cartilage. With time, ossification centers appear in the epiphyses and the epiphyseal growth plates form. The ultrasound characteristics of cartilage differ from those of calcified bone. For example, the velocity of an ultrasound wave through cartilage is $1,700 \mathrm{~m} / \mathrm{sec}$, while its velocity through calcified bone is $2,200-4,500 \mathrm{~m} / \mathrm{sec}$, depending on the type of bone. With the appearance of the centre of ossification and its expansion, the velocity of the wave through the epiphysis will increase until the completion of growth.

The results of this study show good correlations between the data obtained by SBA and both the RUS method and the CASMAS method, but in some cases there were large differences between them. Caution is required because the results sometimes differ according to the measurement position of the wrist. Exact positioning of the wrist during the scanning process is necessary to avoid mistakes in evaluation. This is one of the critical features of measurement with the BonAge system.

In this study, we chose the Caucasian condition in the reference database of the SBA system. It is reported that Japanese bone maturation of the ulnar bone is different from that of Caucasians (4). The details of the SBA algorithm used to calculate bone age are unknown, SBA = $\mathrm{K}_{1}{ }^{\star} \mathrm{SOS}^{\mathrm{C} 1}+\mathrm{K}_{2}{ }^{\star} \mathrm{D}^{\mathrm{C} 2}+\mathrm{K}_{3}\left(\mathrm{~K}_{1}, \mathrm{~K}_{2}, \mathrm{~K}_{3}, \mathrm{C} 1, \mathrm{C} 2\right.$ are constants), with regard to its use in Japanese subjects, further assessments for race, sex, diseases, and age is needed. Longitudinal comparison between the data obtained by SBA and both the RUS method and the CASMAS method are also needed.

SBA offers many advantages, including the ability to make repeated measurements, the absence of any need for interpretation experience or calculations, the ability to make bone age measurements conveniently and rapidly (about 5 min), the absence of x-ray exposure and in consequence that x-ray machines are not needed, and its usefulness for outpatient practice to measure bone ages in children aged 5 to $18 \mathrm{yr}$. Furthermore, in addition to the measurement of subjects' height, weight and age, SBA may provide more information for the development status of healthy children and young athlete.

\section{References}

1. Greulich WW, Pyle SI. Radiographic atlas of skeletal development of the hand and wrist, 2nd ed. Stanford, California: Stanford University Press; 1959.

2. Tanner JM, Whitehouse RH, Cameron N, Marshall WA, Healy MJ, Goldstein H. Assessment of skeletal maturity and prediction of adult height (TW2 method), 2nd ed. London: Academic Press; 1983.

3. Satoh K, Mitani H. Evaluation of bone maturation with a computer. Clin Pediatr Endocrinol 1999;8(suppl 12):13-6.

4. Tanaka N, Satoh N, Tanaka T, Matsuoka H, Murata $\mathrm{M}$, Anzo M, et al. Comparison of bone age estimation methods with computer aided skeletal maturity assessment system and Tannerwhitehouse 2 method standardized for Japanese children in patients of growth hormone deficiency. J Jpn Pediatr Soc 1998;102:145-8 (in Japanese with English abstract).

5. Castriota-Scanderbeg A, De Micheli V. Ultrasound of femoral head cartilage: a new method of 
assessing bone age. Skeletal Radiol 1995;24:197200.

6. Castriota-Scanderbeg A, Sacco MC, EmbertiGialloreti L, Fraracci L. Skeletal age assessment in children and young adults: comparison between a newly developed sonographic method and conventional methods. Skeletal Radiol 1998;27:271-7. 